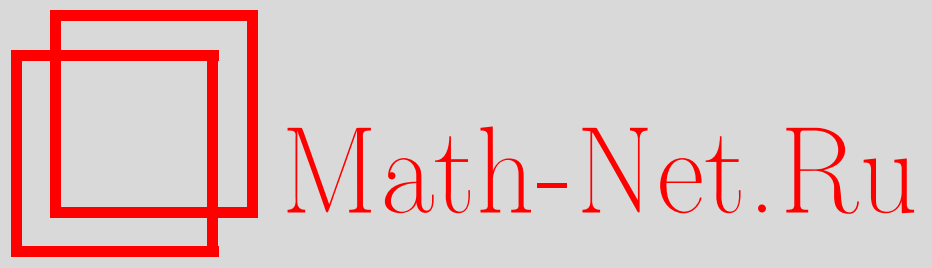

А. А. Боровков, А. А. Могульский, Интегро-локальные предельные теоремы для сумм случайных векторов, включающие большие уклонения. II, Теория вероятн. и ее примен., 2000, том 45, выпуск 1, 5-29

DOI: https://doi.org/10.4213/tvp322

Использование Общероссийского математического портала Math-Net.Ru подразумевает, что вы прочитали и согласны с пользовательским соглашением http://www . mathnet.ru/rus/agreement

Параметры загрузки:

IP : 3.85 .183 .62

26 апреля 2023 г., 15:53:26

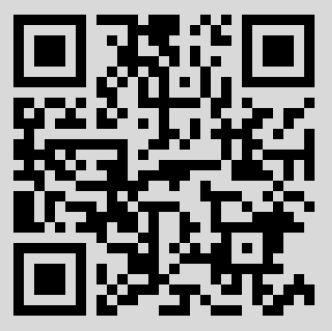


(c) 2000 г. БОРОВКОВ А. А. ${ }^{*}$, МОГУगШ СКИЙ А. А.*

\section{ИНТЕГРО-ЛОКАЛЬНЫЕ ПРЕДЕЛЬНЫЕ ТЕОРЕМЫ ДЛЯ СУММ СЛУЧАЙНЫХ ВЕКТОРОВ, ВКЛЮЧАЮЩИЕ БОЛЬШИЕ УКЛОНЕНИЯ. ІІ ${ }^{1)}$}

Настоящая статья является продолжением работ [1], [2]. Пусть $S(n)=\xi(1)+\cdots+\xi(n)$ есть сумма независимых невырожденных в $\mathbf{R}^{d}$ случайных векторов, распределенных как вектор $\xi$. Предполагается, что функция $\varphi(\lambda)=\mathbf{E} e^{\langle\lambda, \xi\rangle}$ конечна в окрестности некоторой точки $\lambda \in \mathbf{R}^{d}$. Получены асимптотические представления для вероятности $\mathbf{P}\{S(n) \in \Delta(x)\}$ и для функции восстановления $H(\Delta(x))=\sum_{n=1}^{\infty} \mathbf{P}\{S(n) \in \Delta(x)\}$, где $\Delta(x)$ есть куб в $\mathbf{R}^{d}$ с вершиной в точке $x$ и со стороной $\Delta$. При этом в отличие от [1], [2] либо по существу никаких предположений не делается, либо накладываемые условия являются очень слабыми.

Ключевые слова и фразы: большие уклонения, функция уклонений, функция восстановления, интегро-локальная теорема, арифметическое распределение, решетчатое распределение, нерешетчатое распределение.

\section{1. Формулировки результатов}

1.1. Введение. Напомним основные обозначения [1]. Пусть $\boldsymbol{\xi}=$ $\left(\xi_{1}, \ldots, \xi_{d}\right)-$ невырожденный случайный вектор в $d$-мерном евклидовом пространстве $\mathbf{R}^{d}, d \geqslant 1$, с распределением $F$ и преобразованием Лапласа

$$
\varphi(\lambda)=\mathbf{E} e^{\langle\lambda, \xi\rangle}=\int e^{\langle\lambda, x\rangle} F(d x), \quad \lambda \in \mathbf{R}^{d},
$$

где $\langle\lambda, x\rangle=\sum_{i=1}^{d} \lambda_{i} x_{i}$ есть скалярное произведение в $\mathbf{R}^{d}$. Условие невы-

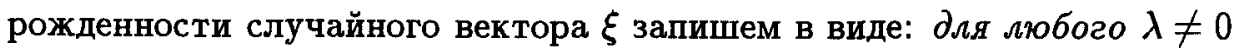

$$
\sup _{t} \mathbf{P}\{\langle\lambda, \xi\rangle=t\}<1
$$

\footnotetext{
${ }^{*}$ Институт математики СО РАН, просп. Академика Коптюга, 4, 630090 Новосибирск, Россия; e-mail: borovkov@math.nsc.ru

1) Работа выполнена при поддержке Российского фонда фундаментальных исследований (проекты № 96-01-01939, № 96-15-96295) и Международного научного фонда (CRDF Cooperating Grants Program, Award RM1-226).
} 
Функцию уклонений, отвечающую вектору $\boldsymbol{\xi}$ (или его распределению $F$ ), обозначим

$$
\Lambda(\alpha)=\sup _{\lambda}\{\langle\lambda, \alpha\rangle-\ln \varphi(\lambda)\}, \quad \alpha \in \mathbf{R}^{d}
$$

Это есть преобразование Лежандра над функцией $A(\lambda)=\ln \varphi(\lambda)$. Функция уклонений играет определяющую роль при описании вероятностей больших уклонений сумм

$$
S(n)=\xi(1)+\cdots+\xi(n)
$$

независимых случайных векторов с общим распределением $F$. Свойства функции уклонений хорошо изучены (см., например, [3]).

Положим $\Phi=\left\{\lambda \in \mathbf{R}^{d}: \varphi(\lambda)<\infty\right\}$ и обозначим через ( $\Phi$ ) внутренность $\Phi$. Множество $\Phi$ всегда непусто $(0 \in \Phi) \cdot$ и выпукло; его внутренность $(\Phi)$ непуста тогда и только тогда, когда выполнено условие Kрамера

$\left(C_{1}\right)$ При всех $\lambda$ из некоторого открытого множества в $\mathbf{R}^{d}$ выполняется

$$
\varphi(\lambda)<\infty .
$$

В настоящей работе мы везде будем предполагать, что условия (1), $\left(C_{1}\right)$ выполнены (если не оговорено противное), хотя многие формулировки остаются справедливыми и вне этих предположений (иногда теряя при этом свою содержательность).

Введем в рассмотрение множества

$$
A^{\prime}=\left\{\alpha: \alpha=A^{\prime}(\lambda)=\operatorname{grad} A(\lambda), \lambda \in \Phi\right\}, \quad \Lambda=\{\alpha: \Lambda(\alpha)<\infty\}
$$

Внутренность $\left(A^{\prime}\right)$ множества $A^{\prime}$ будем считать пустой, если пусто $(\Phi)$, т.е. если не выполнено $\left(C_{1}\right)$. Если выполнено условие $\left(C_{1}\right)$, то функция $\Lambda(\alpha)$ аналитична в области $\left(A^{\prime}\right)$; при этом всегда $\left(A^{\prime}\right) \subseteq \Lambda$. Множество $\Lambda$ всегда выпукло и замкнуто; оно является выпуклой оболочкой носителя распределения $F[3]$. Для $\alpha \in\left(A^{\prime}\right)$ обозначим

$$
\sigma^{2}(\alpha)=\frac{1}{\operatorname{det} \Lambda^{\prime \prime}(\alpha)}, \quad b(\alpha)=\frac{1}{(2 \pi)^{d / 2} \sigma(\alpha)} .
$$

Пусть Z - множество целых чисел на вещественной прямой $\mathbf{R}$. Рacсмотрим также следующие условия (см. [1]).

$\left(C_{2}\right)$ Либо (i) $\limsup \sup _{|s| \rightarrow \infty}|\varphi(i s)|<1$, либо (ii) координаты случайного вектора $\xi$ принимают челбчисленные значения и для некоторого $y \in \mathbf{Z}^{d}$ такого, что $\mathbf{P}\{\xi=y\}>0$, группа, порожденная множеством

$$
\{z: \mathbf{P}\{\xi=y+z\}>0\}
$$


совпадает с множеством $\mathbf{Z}^{d}$ векторов $z=\left(z_{1}, \ldots, z_{d}\right)$ с челочисленньми координатами.

$\left(C_{2}^{(0)}\right)$ Для всяхого вектора $s \in \mathbf{R}^{d}, s \neq 0$, выполняется неравенство $|\varphi(i s)|<1$.

Будем говорить, что вектор $\xi$ имеет нерешетчатое распределение, если он удовлетворяет условию $\left(C_{2}^{(0)}\right)$.

Для точки $\boldsymbol{x}=\left(x_{1}, \ldots, x_{d}\right) \in \mathbf{R}^{d}$ и положительного $\Delta$ обозначим через

$$
\Delta(x)=\left\{y=\left(y_{1}, \ldots, y_{d}\right) \in \mathbf{R}^{d}: x_{i} \leqslant y_{i}<x_{i}+\Delta, i=1, \ldots, d\right\}
$$

куб с основанием в точке $\boldsymbol{x}$ и со стороной $\Delta$.

При выполнении условий $\left(C_{1}\right), \alpha \in\left(A^{\prime}\right),\left(C_{2}\right)$ авторами в [1] были получены так называемые интегро-локальные теоремы для сумм $S(n)$, описывающие асимптотику

$$
\mathbf{P}\{S(n) \in \Delta(x)\}, \quad \alpha=\frac{x}{n} \in\left(A^{\prime}\right), n \rightarrow \infty,
$$

и включающие асимптотические разложения (в случае $\left(C_{2}\right)_{\mathrm{ii}}$ координаты вектора $x$ следует выбирать целочисленными). В [2] аналогичные результаты были получены для функции восстановления

$$
H(\Delta(x))=\sum_{n=1}^{\infty} \mathbf{P}\{S(n) \in \Delta(x)\} .
$$

Цель настоящей работы - получить асимптотические представления для вероятностей (2) и для функиии восстановления (3), отказываясь от условия $\left(C_{2}\right)$ или от всех условии $\left(C_{1}\right), \alpha \in\left(A^{\prime}\right),\left(C_{2}\right)$. Естественно, снятие этих условий ведет к менее точным результатам.

1.2. «Структура» произвольного невырожденного случайного вектора. В этой работе мы не собираемся накладывать какихлибо существенных условий на «структуру» распределения слагаемых $\xi(k)$ (условий типа $\left(C_{2}\right)$ ). С другой стороны, как хорошо известно, асимптотика вероятностей (2) и функции восстановления (3) зависит от этой структуры (см., например, [1], [2]). В связи с этим необходимо выяснить, как может выглядеть эта «структура» в общем случае (для произвольного случайного вектора $\xi$ ). Отметим предварительно, что обе задачи об асимптотическом поведении (2), (3) в известном смысле инвариантны относительно линейных преобразований пространства без сдвига (т.е. преобразований сжатия и поворота). Пусть невырожденная матрица $M$ соответствует такому преобразованию. Тогда знание асимптотик $\mathbf{P}\{S(n) \in x+B\}, H(x+B)$ для борелевского множества $B$ будет означать знание асимптотик $\mathbf{P}\left\{S(n) M \in y+B_{M}\right\}, H_{M}\left(y+B_{M}\right)$ и наоборот; здесь $y=x M, B_{M}=\left\{v: v M^{-1} \in B\right\}, H_{M}(B)=\sum \mathbf{P}\{S(n) M \in B\}$. 
Поэтому мы можем, не ограничивая общности, рассматривать вместо $\xi$ случайный вектор $\zeta=\xi M$ и выбрать матрицу $M$ так, чтобы сделать структуру $\zeta$ наиболее простой.

Оказалось, что в общем случае мы можем выбрать матрицу $M$ так, что координаты $\zeta$ распадаются на следующие три группы.

Лемма 1. Матрицу $M$ можно выбрать так, что все координаты $\zeta_{i}$ можно разбить на три группь

$$
\begin{aligned}
& \zeta^{(1)}=\left(\zeta_{1}, \ldots, \zeta_{m_{1}}\right), \quad \zeta^{(2)}=\left(\zeta_{m_{1}+1}, \ldots, \zeta_{m_{1}+m_{2}}\right) \\
& \zeta^{(3)}=\left(\zeta_{m_{1}+m_{2}+1}, \ldots, \zeta_{d}\right)
\end{aligned}
$$

размерностей $m_{1}, m_{2}, m_{3}$ соответственно, $m_{1}+m_{2}+m_{3}=d$, таким образом, что:

1) первые $m_{1}$ координат составляюо вектор $\zeta^{(1)}=\left(\zeta_{1}, \ldots, \zeta_{m_{1}}\right)$, который имеет арифметическое распределение с шагом 1 по каждой координате, т.е. все координаты принимают челочисленные значения и н.о.д. этих значений по каждой координате равен 1;

2) вторые $m_{2}$ координат составляют вектор

$$
\zeta^{(2)}=a^{(2)}+\bar{\zeta}^{(2)}
$$

где вектор $a^{(2)}=\left(a_{m_{1}+1}, \ldots, a_{m_{1}+m_{2}}\right)$ имеет неслучайные координаmы $a_{i}, 0<a_{i}<1, m_{1}+1 \leqslant i \leqslant m_{1}+m_{2}$, случайный вектор $\bar{\zeta}^{(2)}$ имеет арифметическое распределение с шагом 1 по каждой координате $($ как в первой арупne);

3) последние $m_{3}$ координат составляют вектор $\zeta^{(3)}$ с неречетчатым распределением (удовлетворяют условию $\left(C_{2}^{(0)}\right)$ ).

Таким образом, если первые $m_{1}$ координат $\left(\zeta_{1}, \ldots, \zeta_{m_{1}}\right)=\zeta^{(1)}$ имеют арифметическое распределение с шагом 1 , то следующие $m_{2}$ координат $\left(\zeta_{m_{1}+1}, \ldots, \zeta_{m_{1}+m_{2}}\right)=\zeta^{(2)}$ имеют арифметическое распределение со сдвигом $a^{(2)}$.

В дальнейшем мы будем считать, что преобразование $M$ уже сделано и новый вектор $\zeta=\xi M$ для упрошения обозначений мы вновь будем обозначать $\xi$. Таким образом, мы предполагаем, не ограничивая общности, что вектор $\xi$ с самого начала обладает свойствами, описанными в лемме 1.

Отметим теперь, что при изучении асимптотики вероятностей (2) вторую группу координат можно объединить с первой, рассмотрев для этого случайные векторы $\xi^{*}=\xi-a$ и их суммы $S^{*}(n)=S(n)-n a$, где $d$-мерный вектор $a$ получен из $a^{(2)}$ добавлением $d-m_{2}$ нулевых координат на соответствуюших местах. Асимптотика вероятностей (2) описана в этом случае в теоремах A, 1. 
При изучении функции восстановления (3) положение со второй группой координат обстоит сложнее. Если мы произведем преобразование сдвига, то получим

$$
H(\Delta(x))=\sum_{n=1}^{\infty} \mathbf{P}\left\{S^{*}(n) \in \Delta(\boldsymbol{x})-n a\right\} .
$$

Предположим на время для простоты, что $m_{2}=d$ (арифметическая и нерешетчатые координаты отсутствуют), и обозначим арифметический случайный вектор $\xi^{*}$ снова через $\xi$. Тогда мы придем к задаче вычисления асимптотики

$$
\sum_{n=1}^{\infty} \mathbf{P}\{S(n) \in \Delta(x)-n a\}=\sum_{n=1}^{\infty} \mathbf{P}\left\{S(n) \in \Delta\left(x^{\prime}\right)\right\}
$$

где $x^{\prime}=x+n a$. Но непосредственно применять теорему 1 (см. ниже) здесь нельзя, так как при $\Delta=1$ положение единичного куба $\Delta\left(x^{\prime}\right)$ относительно целочисленной решетки будет с ростом $n$ постоянно меняться. Это создает ряд трудностей, которые не всегда оказываются существенными, если речь идет о единичных кубах, а не о более мелких, и об асимптотике (3) с точностью до постоянного множителя. Более подробное обсуждение вопросов, связанных с присутствием второй группы координат, содержится в замечании после теоремы 3. При этом законченных собирательных результатов при произвольном $a \neq 0$ получить пока не удается. Поэтому в основных утверждениях, касающихся функции восстановления (теорема 3) мы будем считать для простоты, что вторая группа отсутствует, т.е. $m_{2}=0$.

Итак, при изучении асимптотического поведения вероятностей $\mathbf{P}\{S(n) \in \Delta(x)\}$ и функции восстановления $H(\Delta(x))$ мы будем, не ограничивал общности, считать, что произвольный невырожденный случайный вектор $\xi$ с помощью линейного преобразования приведен $к$ виду, описанному в лемме 1. При этом в первом случае (при изучении вероятностей $\mathbf{P}\{S(n) \in \Delta(x)\})$ мы будем, не ограничивая общности, считать, что выполняется $m_{2}=0$, т.е. отсутствует вторая группа координат; во втором случае (при изучении функции $H(\Delta(x))$ ) мы тоже будем предполагать, что $m_{2}=0$, однако здесь это равенство будет уже условием, несколько ограничивающим общность.

Более точное описание и обоснование приведенной выше классификации и доказательство леммы 1 дано в пп. 2.1, 2.2.

Итак, в дальнейшем изложении, касающемся предельных теорем, случайный невырожденный вектор $\xi$ имеет структуру, описанную леммой 1 , где $m_{2}=0$. В этом случае важной характеристикой распределения случайного вектора $\xi$ является число $m=m_{1}$ арифметических координат. 
1.3. Предельные теоремы для сумм. В этом разделе в соответствии с соглашением п. 1.2, мы будем считать, что вектор $\xi^{(1)}=$ $\left(\xi_{1}, \ldots, \xi_{m}\right)$ является арифметическим с шагом 1 по каждой координате, а вектор $\xi^{(3)}=\left(\xi_{m+1}, \ldots, \xi_{d}\right)$ является нерешетчатым. Обозначим

$$
\mathbf{R}^{d}(m)=\mathbf{Z}^{m} \times \mathbf{R}^{d-m},
$$

так что $\mathbf{R}^{d}(0)=\mathbf{R}^{d}$ и для $1 \leqslant m \leqslant d$

$$
\mathbf{R}^{d}(m)=\left\{x=\left(x_{1}, \ldots, x_{d}\right) \in \mathbf{R}^{d}: x_{i} \in \mathbf{Z}, 1 \leqslant i \leqslant m ; x_{i} \in \mathbf{R}, m<i \leqslant d\right\} .
$$

Очевидно, что при сделанном выше соглашении выполняется $\mathbf{P}\{\boldsymbol{\xi} \epsilon$ $\left.\mathbf{R}^{d}(m)\right\}=1$. Поэтому при изучении вероятностей $\mathbf{P}\{S(n) \in \Delta(x)\}$ можно рассматривать лишь $x \in \mathbf{R}^{d}(m)$, а вместо куба $\Delta(x)$ рассматривать «вырожденный» куб $\Delta_{0}^{(m)}$, у которого некоторые стороны равны 0 :

$$
\begin{aligned}
\Delta_{0}^{(m)}(\boldsymbol{x})=\left\{y \in \mathbf{R}^{d}:\right. & y_{j}=x_{j} \text { при } j=1, \ldots, m \\
& \left.x_{j} \leqslant y_{j}<x_{j}+\Delta \text { при } j=m+1, \ldots, d\right\} .
\end{aligned}
$$

Для целого $r, 0 \leqslant r \leqslant d$, вектора $\lambda=\left(\lambda_{1}, \ldots, \lambda_{d}\right) \in \mathbf{R}^{d}$ п вешественного $\Delta>0$ обозначим

$$
\theta^{(r)}(\Delta, \lambda)=\prod_{i=r+1}^{d} \Delta^{-1} \int_{0}^{\Delta} e^{-v \lambda_{i}} d v-1, \quad 0 \leqslant r \leqslant d-1, \quad \theta^{(d)}(\Delta, \lambda)=0 .
$$

Следующая теорема является следствием теоремы 1 в [1].

Теорема А. Пусть выполнено условие $\left(C_{2}\right)$, т.е. челое $m$ равно либо 0, либо $d$, и в случае $m=0$ выполнено условие $\left(C_{2}\right)_{\mathrm{i}}$. Тогда для любого компакта $K \subseteq\left(A^{\prime}\right)$, любого положительного иисла $\delta$, любого неотрицательного $k$ при $\alpha=x / n$ выполняется

$$
\begin{aligned}
\mathbf{P} & \left\{S(n) \in \Delta_{0}^{(m)}(x)\right\} \\
& =\frac{\Delta^{d-m} b(\alpha)}{n^{d / 2}} e^{-n \Lambda(\alpha)}\left(1+\theta^{(d-m)}(\Delta, \lambda(\alpha))+\varepsilon\left(n, \alpha_{\imath} \Delta\right)\right),
\end{aligned}
$$

$2 \partial e$

$$
\lim _{n \rightarrow \infty} \sup _{\alpha \in K, x \in \mathbf{R}^{d}(m)} \sup _{n^{-k} \leqslant \Delta \leqslant \delta}|\varepsilon(n, \alpha, \Delta)|=0 .
$$

Не вызывает сомнения, что теорема А сохранится и при произвольном $m$, лежащем между 0 и $d$, и при дополнительном условии $\left(C_{2}\right)_{i}$ для вектора $\xi^{(3)}=\left(\xi_{m+1}, \ldots, \xi_{d}\right)$. Это соответствует случаю, когда случайный вектор $\xi$ имеет как решетчатые, так и нерешетчатые координаты. Доказательство при этом потребует, конечно, модификаций, но все они укладываются в рамки существующих доказательств, так как основные 
условия, необходимые для работы с формулами обращения, для решетчатой и нерешетчатой частей вектора $\xi$ выполнены.

Одним из главных утверждений настоящей заметки является следуюшая теорема (условия вида $\left(C_{2}\right)$ здесь не предполагагтся).

Теорема 1. Для любого фиксированного $\Delta>0$ и любого компакma $K \subset\left(A^{\prime}\right)$ найдутся челое число $n_{0}<\infty$ и положительные числа $b_{1}(\Delta, K), b_{2}(\Delta, K), 0<b_{1}(\Delta, K) \leqslant b_{2}(\Delta, K)<\infty$, зависяшие только от своих аргументов и от распределения $F$, такие, что для $n \geqslant n_{0}$, $\alpha=x / n \in K, x \in \mathbf{R}^{d}(m)$ выполняется

$$
\mathbf{P}\left\{S(n) \in \Delta_{0}^{(m)}(x)\right\}=\frac{\Delta^{(d-m)} b(\Delta, n, \alpha)}{n^{d / 2}} e^{-n \Lambda(\alpha)},
$$

где $b_{1}(\Delta, K) \leqslant b(\Delta, n, \alpha) \leqslant b_{2}(\Delta, K)$.

Отметим, что константы $b_{1}(\Delta, K), b_{2}(\Delta, K)$ в теореме 1 не зависят от $n$ и $\alpha \in K$, но могут зависеть от параметра $\Delta$. Конечно, выделение множителя $\Delta^{d-m}$ в этом случае теряет, вообще говоря, свой смысл; мы оставляем этот множитель для сохранения единой формы с теоремой $\mathrm{A}$ и в силу того, что в широких условиях константы $b_{1}(\Delta, K), b_{2}(\Delta, K)$ оказываются отделенными от 0 и $\infty$ для всех $\Delta>0$.

Таким образом, в отличие от теоремы $A$, где найдена точная асимптотика в интегро-локальной теореме для сумм $S(n)$, в теореме 1 и следствии 1 (см. далее) при отсутствии условия $\left(C_{2}\right)$ получена асимптотика лишь с точностью до константы.

Остается открытым вопрос:

$\left(Q_{1}\right)$ При каких дополнительных условиях при выполнении соглащения $m_{2}=0$ существует предел $\lim _{n \rightarrow \infty} b(\Delta, n, \alpha)=b(\alpha)+\theta^{(d-m)}(\Delta, \alpha)$ прu $\alpha \in\left(A^{\prime}\right)$ u $\Delta>0$ ?

Заметим, что в одномерном случае соглапгение $m_{2}=0$ означает, что выполнено либо условие $\left(C_{2}\right)_{\text {ii }}$ (когда $m=d=1$ ), либо условие $\left(C_{2}^{(0)}\right)$ (когда $m=d-1=0$ ). В одномерном случае $d=1$ ответ на поставленный вопрос известен: дополнительные условия не требугтся. Этот результат следует из теоремы А в случае $m=1$ и из теоремы 1 в [10] и теоремы 1 в [11] в случае $m=0$. Приведем здесь соответствуюшее утверждение, основанное на процитированных выше теореме А и теореме 1 в [11], которое часто бывает полезно:

Теорема 1А. Пусть $d=1$. Тогда для любого компакта $K \subseteq\left(A^{\prime}\right)$ и любого $\Delta>0$ при $\alpha=x / n \in K$ справедливо

$$
\begin{aligned}
& \mathbf{P}\left\{S(n) \in \Delta_{0}^{(m)}(x)\right\} \\
& \quad=\frac{\Delta^{(1-m)}}{(2 \pi n)^{1 / 2} \sigma(\alpha)} e^{-n \Lambda(\alpha)}\left(1+\theta^{(1-m)}(\Delta, \lambda(\alpha))+\varepsilon(n, \alpha, \Delta)\right),
\end{aligned}
$$

zde $\lim _{n \rightarrow \infty} \sup _{\alpha \in K, x \in \mathbf{R}^{d}(m)}|\varepsilon(n, \alpha, \Delta)|=0$. 
Существенным «недостатком» теорем $\mathrm{A}, 1,1 \mathrm{~A}$ является наличие в них условия $\alpha \in K \subseteq\left(A^{\prime}\right)$, которое ограничивает в ряде случаев (когда $A^{\prime}$ не совпадает с $\mathbf{R}^{d}$ и при этом величина $\xi$ не ограничена) область рассматриваемых уклонений.

Мы перейдем теперь к «грубой интегро-локальной теореме» (локальному принципу больших уклонений), в которой на область рассматриваемых уклонений $\alpha=x / n$ ограничений по существу не накладывается. Тут нам понадобится условие

$\left(C_{1}^{(0)}\right)$ При $\lambda$ из некоторой окрестности точки $\lambda_{0}=0$ выполняется

$$
\varphi(\lambda)<\infty .
$$

Теорема 2 (локальный принцип больших уклонений). Для любого фиксированного $\Delta>0$ и любого компакта $K \subset(\Lambda)$ для $\alpha=x / n \in K$

$$
\frac{1}{n} \ln \mathbf{P}\left\{S(n) \in \Delta_{0}^{(m)}(x)\right\}=-\Lambda(\alpha)+\varepsilon(n, \Delta, \alpha),
$$

где $\lim _{n \rightarrow \infty} \sup _{\alpha \in K, x \in \mathbf{R}^{d}(m)}|\varepsilon(n, \Delta, \alpha)|=0$. Утверждение (9) сохражится и в случае, когда условие $\left(C_{1}\right)$ не выполнено; в этом слуиае $\Lambda(\alpha) \equiv 0$, и утверждению (9) можно придать вид: для любого фиксированного $\Delta>0$ и любого компакта $K \subset \mathbf{R}^{d} \partial \Omega я \alpha=x / n \in K$

$$
\lim _{n \rightarrow \infty} \sup _{\alpha \in K, x \in \mathbf{R}^{d}(m)} \frac{1}{n} \ln \mathbf{P}\left\{S(n) \in \Delta_{0}^{(m)}(x)\right\}=0 .
$$

Если выполнено условие $\left(C_{1}^{(0)}\right)$, то для любого $M<\infty$ найдется $T=T(M)<\infty$ maxoe, umo

$$
\limsup _{n \rightarrow \infty} \frac{1}{n} \ln \mathbf{P}\{|S(n)| \geqslant n T\} \leqslant-M .
$$

Как мы уже отмечали, $\mathbf{P}\left\{n^{-1} S(n) \notin \Lambda\right\}=0$; таким образом, coomношение

$$
\lim _{n \rightarrow \infty} \frac{1}{n} \ln \mathbf{P}\left\{S(n) \in \Delta_{0}^{(m)}\left(x_{n}\right)\right\}=-\Lambda(\alpha)
$$

для $x_{n} \in \mathbf{R}^{d}(m), x_{n} / n \rightarrow \alpha$ верно, по существу, без каких бы то ни было ограничений.

Из утверждения (9) теоремы следует обычный приниип больших уклонений для нормированных сумм $S(n) / n$ (см., например, [3]; тут никаких предположений о структуре вектора $\xi$ можно не делать):

(a) для любых ограниченных замкнутых множеств $B$

$$
\limsup _{n \rightarrow \infty} \frac{1}{n} \ln \mathbf{P}\left\{\frac{S(n)}{n} \in B\right\} \leqslant-\inf _{\alpha \in B} \Lambda(\alpha) ;
$$


(b) для любых открытых множеств $B$

$$
\liminf _{n \rightarrow \infty} \frac{1}{n} \ln \mathbf{P}\left\{\frac{S(n)}{n} \in B\right\} \geqslant-\inf _{\alpha \in B} \Lambda(\alpha) .
$$

В одномерном случае $d=1$ соотношение (12) верно для любых замкнутых множеств $B$. В случае $d \geqslant 2$ это, вообще говоря, не так, о чем свидетельствует пример в [12]. Если же выполнено условие $\left(C_{1}^{(0)}\right)$, то соотношение (12) верно для любых замкнутых множеств $B$ в пространстве $\mathbf{R}^{d}$ любой размерности $d \geqslant 1$ (см., например, [3]).

Вернемся теперь к интегро-локальным теоремам 1, 1А и покажем, как из этих теорем получить утверждения интегрального типа.

Назовем множества

$$
\begin{aligned}
B^{(\varepsilon)} & =\left\{x \in \mathbf{R}^{d}: \text { существует вектор } y \in B \text { такой, что }|x-y|<\varepsilon\right\} ; \\
B^{(-\varepsilon)} & =\{x \in B: \text { для всякого вектора } y \notin B \text { выполняется }|x-y|>\varepsilon\}
\end{aligned}
$$

соответственно внешней и внутренней $\varepsilon$-окрестностями множества $B$.

Непосредственным следствием из теоремы 1 является следующее утверждение (тут также никаких предположений о структуре вектора $\xi$ можно не делать).

Следствие 1. Для некоторого $\delta \geqslant 0, \partial \Omega я$ любых $\Delta \geqslant \delta$ и компакта $K \subset\left(A^{\prime}\right)$ найдутся челое число $n_{0}=n_{0}(\Delta, K)<\infty$ и положительные числа $b_{1}, b_{2}, 0<b_{1} \leqslant b_{2}<\infty$, такие, что для всех $n \geqslant n_{0}$ и любого измеримого множества $B \subseteq K$ выполняется

$$
\begin{aligned}
\frac{b_{1}}{n^{d / 2}} \int_{B^{(-\Delta / n)}} e^{-n \Lambda(\alpha)} d \alpha & \leqslant \mathbf{P}\{S(n) \in n B\} \\
& \leqslant \frac{b_{2}}{n^{d / 2}} \int_{B^{(\Delta / n)}} e^{-n \Lambda(\alpha)} d \alpha .
\end{aligned}
$$

Eсли дополнительно выполнено условие $\left(C_{2}^{(0)}\right)$, то (14) верно для любых $\Delta>0$.

Если множество $B$ таково, что для любого $\Delta>0$ найдутся константы $c, C, 0<c \leqslant C<\infty$, такие, что для всех достаточно больших $n$ выполняется

$$
c \int_{B^{(\Delta / n)}} e^{-n \Lambda(\alpha)} d \alpha \leqslant \int_{B} e^{-n \Lambda(\alpha)} d \alpha \leqslant C \int_{B^{(-\Delta / n)}} e^{-n \Lambda(\alpha)} d \alpha,
$$

то для этого $B$ при всех достаточно больших $n$ справедливо соотношение

$$
\frac{b_{1}}{C n^{d / 2}} \int_{B} e^{-n \Lambda(\alpha)} d \alpha \leqslant \mathbf{P}\{S(n) \in n B\} \leqslant \frac{b_{2}}{c n^{d / 2}} \int_{B} e^{-n \Lambda(\alpha)} d \alpha .
$$

Условие (15) для множества $B$ будет выполнено, если это множество в окрестности «наиболее вероятной» точки $\alpha_{0} \in B$ (т.е. такой, что 
$\Lambda\left(\alpha_{0}\right)=\inf _{\alpha \in B} \Lambda(\alpha)$; при $\Lambda\left(\alpha_{0}\right)>0$ точка $\alpha_{0}$ лежит на границе $\left.B\right) \ll т е-$ лесно» и имеет «не очень рыхлую» границу.

Таким образом, для достаточно широкого класса множеств $B$ асимптотика вероятности $\mathbf{P}\{S(n) \in n B\}$ найдена с точностью до константы в форме (16). Этот результат в известном смысле неулучшаем: в рассматриваемых условиях $(1),\left(C_{1}\right)$ даже для таких «простых» множеств $B$, как полупространство $\{\alpha:\langle\alpha, \lambda\rangle \geqslant c\}$, соотношение (16) нельзя заменить более точным утверждением

$$
\mathbf{P}\{S(n) \in n B\}=\frac{b+o(1)}{n^{d / 2}} \int_{B} e^{-n \Lambda(\alpha)} d \alpha
$$

для подходящей константы $b$ (так может обстоять дело, например, в решетчатом одномерном случае для множества $B=\{\alpha: \alpha \geqslant \sqrt{2}\}$ ). Отметим еще, что результат (16) для более узкого класса $\mathscr{D}$ борелевских множеств $B$, представимых в виде $B=A_{1} \backslash A_{2}$, где $A_{1}, A_{2}$ - выпуклые множества, был получен в [4]. Для более узкого класса уклонений $\alpha_{0}=\mathbf{E} \xi+o(1)$ и для класса множеств $\mathscr{D}$ в работах [4], [5] доказана эта более точная, чем (16), формула (17) (см. также близкие результаты в [6]-[8]). Отметим еще, что результат (17) невозможно вывести непосредственно из теоремы 1 , в которой изначально найдена асимптотика с точностью до константы и которая тем самым ориентирована на изучение самой общей ситуации.

1.4. Предельные теоремы для функции восстановления. Обратимся теперь к изучению «многомерной» функции восстановления

$$
H(A)=\sum_{n=1}^{\infty} \mathbf{P}\{S(n) \in A\}, \quad A \subseteq \mathbf{R}^{d} .
$$

В этом разделе, в соответствии с соглашением, принятым в п. 1.2, мы снова будем считать, что случайный вектор $\xi$ имеет структуру, определенную леммой 1 , и группа арифметических координат со сдвигом отсутствует $\left(m_{2}=0\right)$. Напомним, что в этом случае первая группа состоит из $m=m_{1}$ арифметических координат, а остальные $d-m$ координат образуют нерешетчатый вектор.

В работе [2] авторы получили в условиях $\left(C_{1}\right),\left(C_{2}\right)$ асимптотические разложения для функции

$$
H(\Delta(x)), \quad|x| \rightarrow \infty .
$$

В настоящей работе получен несколько менее точный результат, но без привлечения условия $\left(C_{2}\right)$.

В описании асимптотики функции восстановления определяющую роль играет так называемая вторая функция уклонений

$$
D(\alpha)=\inf _{s>0} \frac{\Lambda(s \alpha)}{s}
$$


которая была введена и достаточно полно изучена авторами в [2]. В частности, функция $D(\alpha)$ выпукла: $\partial л я ~ \alpha_{1}, \alpha_{2} \in \mathbf{R}^{d}, p_{1}, p_{2} \geqslant 1, p_{1}+p_{2}=1$,

$$
D\left(p_{1} \alpha_{1}+p_{2} \alpha_{2}\right) \leqslant p_{1} D\left(\alpha_{1}\right)+p_{2} D\left(\alpha_{2}\right),
$$

и линейна по любому направлению: для $\alpha \in \mathbf{R}^{d}, t \geqslant 0$ имеем $D(t \alpha)=$ $t D(\alpha)$, и при этом $D(t \mathbf{E} \xi)=t D(\mathbf{E} \xi)=0$.

Обозначим

$$
E=\left\{\lambda \in \mathbf{R}^{d}: \varphi(\lambda) \leqslant 1\right\}, \quad D=\left\{\alpha \in \mathbf{R}^{d}: \alpha \neq 0, D(\alpha)<\infty\right\}
$$

Множество $E$ не пусто и выпукло; функция $D(\alpha)$ допускает следующее представление:

$$
D(\alpha)=\sup _{\lambda \in E}\langle\lambda, \alpha\rangle=\sup _{\lambda \in \partial E}\langle\lambda, \alpha\rangle=\langle\mu(\alpha), \alpha\rangle,
$$

где вектор $\mu(\alpha)$ лежит на гражиче $\partial E$ множества $E$ (см. [2]). Определим далее функцию $s(\alpha)>0$ как значение $s$, при котором достигается inf в (19):

$$
D(\alpha) \equiv \frac{\Lambda(s(\alpha) \alpha)}{s(\alpha)} .
$$

Для $\alpha \in D$ определим подмножество $E_{\alpha} \subseteq E$ :

$$
E_{\alpha}=\{\lambda \in E:\langle\lambda, \alpha\rangle=D(\alpha)\}
$$

Тогда множество

$$
\left(D_{\Phi}\right)=\left\{\alpha \in D: E_{\alpha} \subseteq(\Phi)\right\}
$$

является сектором в $\mathbf{R}^{d}$ (ср. с определением множества $\left(A^{\prime}\right)$ ); оно играет роль множества регулярности для функции $D(\alpha)$, аналогично той, какую играет множество $\left(A^{\prime}\right)$ для функции $\Lambda(\alpha)$. В частности, если $E \subseteq(\Phi)$, то введенный сектор совпадает со всем пространством: $\left(D_{\Phi}\right)=\mathbf{R}^{d}$.

Проекцию $\left(D_{\Phi}\right)$ на единичную сферу $\mathbf{R}^{d}$ обозначим

$$
\left(D_{\Phi}^{(1)}\right)=\left\{\alpha \in\left(D_{\Phi}\right):|\alpha|=1\right\} \text {. }
$$

Аналогом теоремы А является следующая теорема В, которая вытекает из теорем 5,6 в [2].

Теорема В. Пусть выполнено условие $\left(C_{2}\right)$, т.е. челое число $m$ равно либо 0, либо $d$, и в случае $m=0$ выполнено условие $\left(C_{2}\right)_{\mathrm{i}}$. Пусть $t=|x|, v=x /|x|$.

I. Ecлu $\mathbf{E} \xi \neq 0$, mо для мюбого $\delta>0$, мюбого $k>0$, мюбого компакта $K \subseteq\left(D_{\Phi}^{(1)}\right)$ выполняется

$$
H\left(\Delta_{0}^{(m)}(x)\right)=\frac{h(v) \Delta^{d-m}}{t^{(d-1) / 2}} e^{-t D(v)}\left(1+\theta^{(d-m)}(\Delta, \mu(v))+\varepsilon(x, \Delta)\right)
$$


$2 \partial e$

$$
\begin{aligned}
& \lim _{t \rightarrow \infty} \sup _{v \in K, x \in \mathbf{R}^{d}(m)} \sup _{t^{-k} \leqslant \Delta \leqslant \delta}|\varepsilon(x, \Delta)|=0 \\
& h(v)=\frac{\left(v \Lambda^{\prime \prime}(s(v) v) v^{T}\right)^{-1 / 2}}{\sigma^{2}(s(v) v)(2 \pi)^{(d-1) / 2} s(v)^{(d+3) / 2}} .
\end{aligned}
$$

II. Если выполнено условие $\left(C_{1}^{(0)}\right), \mathbf{E} \xi=0, d \geqslant 3, B^{2}=\mathbf{E} \xi^{T} \xi$, то для любых $\delta>0, k>0$ и компакта $K \subseteq\left(D_{\Phi}^{(1)}\right)$ выполняется

$$
H(\Delta(x))=\frac{q(v) \Delta^{d}}{t^{d-2}}(1+\varepsilon(x, \Delta)),
$$

zдe

$$
\begin{gathered}
\lim _{|x| \rightarrow \infty} \sup _{v \in K, x \in \mathbf{R}^{d}(m)} \sup _{t^{-k} \leqslant \Delta \leqslant \delta}|\varepsilon(x, \Delta)|=0, \\
q(v)=\frac{1}{\sigma^{2}(0)(2 \pi)^{d / 2}} \int_{0}^{\infty} e^{-v B^{-1} v^{T} /(2 u)} \frac{d u}{u^{d / 2}} .
\end{gathered}
$$

Не вызывает сомнения, что теорема В сохранится и при произвольном $m$, лежащем между 0 и $d$, и при дополнительном условии $\left(C_{2}\right)_{\mathrm{i}}$ для вектора $\left(\xi_{m+1}, \ldots, \xi_{d}\right)$ (см. замечание, приведенное после теоремы A).

Одно из основных утверждений настоящей работы, касающееся функции уклонений, состоит в следуюшем (условия типа $\left(C_{2}\right)$ здесъ не предполагаются).

Теорема 3. $\Pi y c m b ~ t=|x|, v=x /|x|$.

I. Если $\mathbf{E} \xi \neq 0$, то для любого $\Delta>0$, любого компакта $K \subseteq$ $\left(D_{\Phi}^{(1)}\right)$ найдутся положительные числа $t_{0}<\infty, h_{1}(\Delta, K), h_{2}(\Delta, K), 0<$ $h_{1}(\Delta, K) \leqslant h_{2}(\Delta, K)<\infty$, такие, ито $n$ рu $t \geqslant t_{0}, v \in K, x \in \mathbf{R}^{d}(m)$ выполняется

$$
H\left(\Delta_{0}^{(m)}(x)\right)=\frac{\Delta^{d-m} h(\Delta, x)}{t^{(d-1) / 2}} e^{-t D(v)},
$$

əde

$$
h_{1}(\Delta, K) \leqslant h(\Delta, x) \leqslant h_{2}(\Delta, K)
$$

II. Eсли $\mathbf{E} \xi=0, d \geqslant 3$, то для любого $\Delta>0$, любого компакта $K \subseteq\left(D_{\Phi}^{(1)}\right)$ найдутся положительные числа $t_{0}<\infty, q_{1}(\Delta, K), q_{2}(\Delta, K)$, $0<q_{1}(\Delta, K) \leqslant q_{2}(\Delta, K)<\infty$, maкuе, uто при $t \geqslant t_{0}, v \in K, x \in \mathbf{R}^{d}(m)$ выполняется

$$
H\left(\Delta_{0}^{(m)}(x)\right)=\frac{\Delta^{d-m} q(\Delta, x)}{t^{d-2}},
$$

zde

$$
q_{1}(\Delta, K) \leqslant q(\Delta, x) \leqslant q_{2}(\Delta, K)
$$


Как и константы $b_{1}, b_{2}$ в теореме 1 , константы $h_{1}, h_{2}, q_{1}, q_{2}$ в теореме 3 зависят, вообще говоря, от параметра $\Delta$.

Сделаем теперь ряд замечаний, касающихся случая $m_{2}>0$ (см. п. 1.2), когда среди координат вектора $\xi$ есть группа $\xi^{(2)}$ объема $m_{2} \geqslant 1$ арифметических координат «со сдвигом» $a^{(2)}=$ $\left(a_{m_{1}+1}, \ldots, a_{m_{1}+m_{2}}\right), 0<a_{i}<1, i=m_{1}+1, \ldots, m_{1}+m_{2}$.

Пусть для простоты $m_{2}=d$. Как было отмечено в п. 1.2 , задача оценивания асимптотики $H(\Delta(x))$ в этом случае сводится к задаче об асимптотике

$$
\sum_{n=1}^{\infty} \mathbf{P}\left\{S(n) \in \Delta\left(x^{\prime}\right)\right\}
$$

для арифметического случайного вектора $\xi$, где $x^{\prime}=x-n a$. Непосредственно применять тут теорему 1 нельзя, так как положение куба $\Delta\left(x^{\prime}\right)$ относительно решетки $\mathbf{Z}^{d}$ при разных значениях $n$ меняется и при $\Delta<1$ вероятности $\mathbf{P}\left\{S(n) \in \Delta\left(x^{\prime}\right)\right\}$ могут обрашаться в 0 . Но если $\Delta=1$, то существует единственная точка $x^{(0)} \in \Delta\left(x^{\prime}\right)$ с целочисленными координатами, и мы можем, согласно теореме 1 , найти асимптотику

$$
\mathbf{P}\left\{S(n)=x^{(0)}\right\}=\mathbf{P}\left\{S(n) \in \Delta\left(x^{\prime}\right)\right\} .
$$

Если теперь в полученном соотношении для $\mathrm{P}\left\{S(n)=x^{(0)}\right\}$ в правой части в множителе $b(\alpha) e^{-n \Lambda(\alpha)}$ значение $\alpha=x^{(0)} / n$ заменим на $\alpha^{\prime}=x^{\prime} / n$, то мы получим выражение, которое отличается от исходного положительным множителем, отделенным от 0 и $\infty$, и которое совпадает с тем, которое стоит в правой части теоремы 1 . Таким образом, при $\Delta=1$ утверждение теоремы 1 сохранится при любом $x$ и, стало быть, с точки зрения утверждений теорем 1,3 группа $m_{2}=d$ арифметических координат со сдвигом обладает тем же свойством, что и группа нерешетчатых координат (при этом $m=m_{1}=0$, и под кубом $\Delta_{0}^{(m)}(x)=\Delta_{0}^{(0)}(x)$ следует понимать куб $\Delta(x))$.

Поэтому в общем случае при $0 \leqslant m_{2} \leqslant d$ и $n p u \Delta \geqslant 1$ утверждения теорем 1, 3 остаются справедливьми (число т при этом сохраняет прежнее значение $\left.m=m_{1}\right)$.

Значительно более сложным является вопрос о том, когда утверждения теорем 1,3 останутся справедливыми для более «мелких кубов» при $\Delta<1$. Тут существенную роль начинают играть арифметические свойства координат $a_{i}, m_{1}+1 \leqslant i \leqslant m_{1}+m_{2}$, вектора «сдвигов» $a^{(2)}$.

Пусть вновь для простоты $m_{2}=d$. Если все $a_{i}$ рациональны, то можно определить «более мелкую», чем $\mathbf{R}^{d}(d)=\mathbf{Z}^{d}$, решетку $L$, которая есть группа по операции сложения в $\mathbf{R}^{d}$, порожденная множеством $\mathbf{Z}^{d}+a$. На этой решетке $L$ остается справедливой версия теоремы В о точной асимптотике $H(\Delta(\boldsymbol{x}))$ для арифметического случая, но с меньшим постоянным множителем, равным $l^{-1}$, где $l$ есть число точек решетки $L$ в 
Боровков А. А., Могульский $A . A$.

единичном кубе $\Delta(0)$. Пусть, скажем, $d=m_{2}=1$ п $a_{1}=\frac{1}{2} ;$ тогда мы будем иметь дело с «периодическим блужданием» $(S(2 n)$ всегда целое), а «более мелкая» решетка $L$ имеет вид $L=\frac{1}{2} \mathbf{Z}^{1}$. В этом случае утверждение теоремы В для $x \in L$ сохранится с правой частью, помноженной на $\frac{1}{2}$.

Таким образом, в общем случае, когда $d \geqslant m_{2}>0$ и координаты $a_{i}$ вектора $a^{(2)}$ рациональны, можно сиитать $m=m_{1}+m_{2}$, u утверждение теоремы 3 сохранится для любого $\Delta>0 u x \in L$, где $L$ есть группа по операчии сложения в $\mathbf{R}^{d}$, порожденная множеством $\mathbf{Z}^{d}(m)+a$, вектор $a \in \mathbf{R}^{d}$ получается из вектора $a^{(2)}$ присоединением нулевьх координат на соответствуюиих местах.

Если все $a_{i}$ иррациональны и «несоизмеримы», так что дробные части $\left\{n a^{(2)}\right\}$ чисел $n a^{(2)}$ при $m_{2}=d$ равномерно заполняют единичный куб $\Delta(0)$ (отношение числа попаданий элементов последовательности $\left\{k a^{(2)}\right\}, k=1,2, \ldots, n$, в телесное множество $B \subseteq \Delta(0)$ к числу $n$ сходится при $n \rightarrow \infty$ к лебеговой мере $B$ ), то утверждение теоремы 3 сохранится для любого $\Delta>0$ и $x \in \mathbf{R}^{d}$. Отсюда нетрудно получить, что в общем случае $0<m_{2} \leqslant d$ для «несоизмеримых» иррациональных координат $a_{i}, m_{1}+1 \leqslant i \leqslant m_{1}+m_{2}$, утверждение теоремы 3 также останется в силе; но при этом вторую группу из $m_{2}$ координат следует «объединить» с третьей, так что в этом случае $m=m_{1}$.

Если же $a_{i}$ иррациональны и соизмеримы, то описание асимптотики $H(\Delta(x))$ будет более сложным (например, для $d=m_{2}=2, a_{1}=a_{2}=$ $\sqrt{2} / 2$ числа $\left\{n a^{(2)}\right\}$ заполняют диагональ единичного куба).

Таким образом, вопрос об общих условиях, обеспечивающих справедливость теоремы 3 в случае, когда $m_{2} \geqslant 1$ и среди чисел $a_{i}$ есть иррациональныг, остается открытым. Открытым также остается вопрос: при каких дополнительных условиях в теореме 3 существуют пределы

$$
\lim _{|x| \rightarrow \infty, x /|x| \rightarrow v} h(\Delta, x)=h(v), \quad \lim _{|x| \rightarrow \infty, x /|x| \rightarrow v} q(\Delta, x)=q(v)
$$

при фиксированных $v \in\left(D_{\Phi}^{(1)}\right) u \Delta>0$ ?

Очевидно, что ответ на вопрос $\left(Q_{1}\right)$ дает ответ на последний вопрос. Поэтому в одномерном случае $d=1$ никаких дополнительных условий для существования пределов (26) не требуется.

Приведем теперь «грубую интегро-локальную теорему» для функции восстановления $H$, соответствующую части I теоремы 3 и справедливую без привлечения каких-либо соглашений о структуре вектора $\xi$.

Tеорема 4. Пусть $\mathbf{E} \xi \neq 0, t=|\boldsymbol{x}|, v=x /|\boldsymbol{x}|$. Tогда найдеmсл $\Delta_{0} \geqslant 0$ maхое, ито для пюбого $\Delta \geqslant \Delta_{0}$ u для любого компакта 
$K \subseteq\left(D_{\Phi}^{(1)}\right)$ выполняется

$$
\frac{1}{t} \ln H(\Delta(x))=-D(v)+\varepsilon(x, \Delta),
$$

2дe $\lim _{t \rightarrow \infty} \sup _{v \in K}|\varepsilon(x, \Delta)|=0$.

В качестве простого следствия из теоремы 4 получаем интегролокальную теорему для функции восстановления $H$ в форме, которая может быть удобна для приложений.

Следствие 2. Пусть $\mathbf{E} \xi \neq 0$ u $D(\boldsymbol{x})<\infty$ для всех $\boldsymbol{x} \in \mathbf{R}^{d}$. Тогда для некоторого $\Delta_{0} \geqslant 0$ u для любых $\varepsilon>0, \Delta \geqslant \Delta_{0}$ найдется $C<\infty$ такое, что для всех $\boldsymbol{x} \in \mathbf{R}^{d}$ выполняется

$$
\frac{1}{C} e^{-D(x)-\varepsilon|x|} \leqslant H(\Delta(x)) \leqslant C e^{-D(x)+\varepsilon|x|} .
$$

Из теоремы 4 вытекает принцип больших уклонений для функции восстановления, полученный в [2]: $n p u \mathbf{E} \xi \neq 0$

(a) для любых ограниченных замкнутых множеств $B$

$$
\limsup _{t \rightarrow \infty} \frac{1}{t} \ln H(B) \leqslant-\inf _{\alpha \in B} D(\alpha) ;
$$

(b) для любых открытых множеств $B$

$$
\liminf _{t \rightarrow \infty} \frac{1}{t} \ln H(B) \geqslant-\inf _{\alpha \in B} H(\alpha) .
$$

\section{2. Доказательства основных утверждений}

2.1. Минимальная решетка для невырожденного случайного вектора. Для произвольных целого $m, 0 \leqslant m \leqslant d$, невырожденной квадратной матрицы $M=\left(M_{i j}\right)_{i, j=1}^{d}$ и вектора $a=\left(a_{1}, \ldots, a_{d}\right) \in \mathbf{R}^{d}$ введем множество

$$
L_{m}=L_{m}(M, a)=\left\{x \in \mathbf{R}^{d}: x M \in a+\mathbf{R}^{d}(m)\right\},
$$

где, как и прежде, $\mathbf{R}^{d}(m)=\mathbf{Z}^{m} \times \mathbf{R}^{d-m}$. Множество $L_{m}(M, a)$ назовем решеткой (невырожденной) в $\mathbf{R}^{d}$ размерности $m$. В частности, само множество $\mathbf{R}^{d}(m)$ есть решетка в $\mathbf{R}^{d}$ размерности $m$. Заметим, что в определении решетки $L_{m}(M, a)$ можно считать, не ограничивая общности, что $0 \leqslant a_{i}<1$ для $i \in\{1, \ldots, m\}$ и координаты вектора $a$ с номерами $i \in\{m+1, \ldots, d\}$ выбраны нулевыми. Что касается матрицы $M$, то свою роль в определении решетки $L_{m}(M, a)$ играют только столбцы этой матрицы с номерами $i \in\{1, \ldots, m\}$; остальные столбцы можно выбирать произвольным образом, сохраняя при этом невырожденность $M$. 
Множество $L_{m}(M, 0)$ является замкнутой подгруппой (по операции сложения векторов) в пространстве $\mathbf{R}^{d}$, поэтому решетка $L_{m}(M, a)$ является «сдвинутой» замкнутой подгруппой в $\mathbf{R}^{d}$ (см. (32) ниже). Структура замкнутых подгрупп в $\mathbf{R}^{d}$ описана, например, в [9] (теорема 21.2):

Пусть $\Gamma-$ замкнутая подеруппа в $\mathbf{R}^{d}$. Пусть $r-$ максимальнал размерность линейных подпространств в $\Gamma$ и $r+m \leqslant d-$ максимальное иисло линейно независимых векторов в $Г$. Тогда существуют такие линейно независимые векторы $z_{1}, \ldots, z_{m} ; y_{1}, \ldots, y_{\tau} \in \Gamma$, что

$$
\begin{gathered}
\Gamma=\left\{x=k_{1} z_{1}+\cdots+k_{m} z_{m}+t_{1} y_{1}+\cdots+t_{r} y_{r}:\right. \\
\left.k_{i} \in \mathbf{Z}, 1 \leqslant i \leqslant m ; t_{j} \in \mathbf{R}, 1 \leqslant j \leqslant r\right\} .
\end{gathered}
$$

Заметим, что в (30) можно считать, не ограничивая общности, что пары векторов $z_{i}, y_{j}$ ортогональны для всех $i=1, \ldots, m, j=1, \ldots, r$.

Пусть размерность замкнутой подгруппы $\Gamma^{*}$ в $\mathbf{R}^{d}$ совпадает $\mathbf{c} d$, т.е. $m+r=d$. Рассмотрим квадратную невырожденную матрицу

$$
B=\left(z_{1}^{T}, \ldots, z_{m}^{T}, y_{1}^{T}, \ldots, y_{r}^{T}\right),
$$

столбцы которой составлены из координат векторов $z_{1}, \ldots, z_{m}, y_{1}, \ldots, y_{r}$. Можно проверить, что $\Gamma^{*}=L_{m}\left(B^{-1}, 0\right)$ и для любого вектора $a^{*} \in \mathbf{R}^{d}$ справедливо

$$
a^{*}+\Gamma^{*}=L_{m}\left(B^{-1}, a^{*} B^{-1}\right) .
$$

Будем называть решетку $L_{m}=L_{m}(M, a)$ минимальной решеткой, отвечающей невырожденному случайному вектору $\xi$, если

$$
\mathbf{P}\left\{\xi \in L_{m}\right\}=1
$$

и не существует другой решетки $L_{m^{\prime}}\left(M^{\prime}, a^{\prime}\right)$, которая обладала бь свойством (33) и была бы собственным подмножеством решетки $L_{m}(M, a)$.

Ниже, в п. 2.2 мы приведем алгоритм построения минимальной решетки для невырожденного вектора $\xi$ с помощью характеристической функции $f(t)=\varphi(i t)$. Тем самым, будет установлено, что любому невырожденному случайному вектору $\xi$ отвечает его минимальная решетка. Такую минимальную решетку, отвечающую невырожденному вектору $\xi$, будем обозначать $L^{\xi}$, а ее размерность $m$ обозначим $m^{\xi}$.

Нерешетчатому вектору $\xi$ (т.е. удовлетворяюшему условию $\left(C_{2}^{(0)}\right)$ ) отвечает минимальная решетка $L^{\xi}=L_{0}(M, a)=\mathbf{R}^{d}$ размерности 0 .

Будем, следуя [9], называть вектор $\xi$ решетчатым, если найдется решетка $L_{d}=L_{d}(M, a)$ размерности d такал, что

$$
\mathbf{P}\left\{\xi \in L_{d}(M, a)\right\}=1 .
$$


Если решетчатый вектор $\xi$ является невырожденным, то очевидно, что ему будет соответствовать минимальная решетка $L^{\xi}=L_{d}(M, a)$ размерности $d$. Отметим еше, что приведенное выше условие $\left(C_{2}\right)_{\text {iі }}$ можно переписать в виде:

$\left(C_{2}\right)_{\text {iі }}$ Минимальная решетка $L^{\xi}$ совпадает $с \mathbf{Z}^{d}$.

2.2. Доказательство леммы 1. Осушествим построение минимальной решетки для невырожденного вектора $\xi$. Если выполнено условие $\left(C_{2}^{(0)}\right)$, то положим $L^{\xi}=\mathbf{R}^{d}=L_{0}$.

Если условие $\left(C_{2}^{(0)}\right)$ не выполнено, то определено отличное от $\{0\}$ множество

$$
T=T^{\xi}=\left\{t \in \mathbf{R}^{d}:|f(t)|=1\right\} .
$$

Пусть $m$ есть размерность линейного подпространства $\Pi_{1}$, порожденного множеством $T$. Последняя и будет размерностью $m^{\xi}$ решетки $L^{\xi}$. Обозначим $\Pi_{2}$ ортогональное дополнение $\mathbf{~} \Pi_{1}$ и представим случайный вектор $\xi$ в виде суммы

$$
\xi=\xi_{1}+\xi_{2},
$$

где $\xi_{i}$ есть проекция $\xi$ на подпространство $\Pi_{i}, i=1,2$. Очевидно, что вектор $\xi_{1}$ является решетчатым вектором в $\mathbf{R}^{d}$ (вырожденным в $\mathbf{R}^{d}$, если $\Pi_{1} \neq \mathbf{R}^{d}$ ) и при этом невырожденным в $\Pi_{1}$; вектор $\xi_{2}$ является невырожденным нерешетчатым вектором в $\Pi_{2}$.

Пусть Г есть дискретная подгруппа в $\Pi_{1}$, порожденная множеством

$$
\left\{x \in \Pi_{1}: \mathbf{P}\left\{\xi_{1}=x_{0}+x\right\}>0\right\},
$$

где $x_{0} \in \Pi_{1}-$ произвольный вектор в $\mathbf{R}^{d}$ такой, что $\mathbf{P}\left\{\xi_{1}=x_{0}\right\}>0$. В силу лемм 21.4 и 21.6 в [9] эта подгруппа Г является минимальной группой, связанной с $\xi_{1}$, т.е. единственной дискретной подеруппой со следуюшими свойствами: (1) $\mathbf{P}\left\{\xi_{1}=x+\Gamma\right\}=1$ для всякого $x \in \Pi_{1}$ такого, что $\mathbf{P}\left\{\xi_{1}=x\right\}>0 ;(2)$ если $\Gamma_{1}-$ любая замкнутал подеруппа такая, ито $\mathbf{P}\left\{\xi_{1} \in y+\Gamma_{1}\right\}=1$ при некотором $y \in \Pi_{1}$, mо $\Gamma \subseteq \Gamma_{1}$. $\mathrm{B}$ силу теоремы 21.1 в [9] (см. (31) выше) в Г существует ровно $m$ линейно независимых векторов $z_{1}, \ldots, z_{m}$ таких, что

$$
\Gamma=\left\{x=k_{1} z_{1}+\cdots+k_{m} z_{m}: k_{i} \in \mathbf{Z}, i=1, \ldots, m\right\} \text {. }
$$

Выберем, далее, $r=d-m$ линейно независимых векторов $y_{1}, \ldots, y_{r}$ в $\Pi_{2}$ и введем в рассмотрение замкнутую подгруппу размерности $d$

$$
\begin{gathered}
\Gamma^{*}=\left\{x=k_{1} z_{1}+\cdots+k_{m} z_{m}+t_{1} y_{1}+\cdots+t_{r} y_{r}:\right. \\
\left.k_{i} \in \mathbf{Z}, 1 \leqslant i \leqslant m ; t_{j} \in \mathbf{R}, 1 \leqslant j \leqslant r\right\} .
\end{gathered}
$$

Пусть, далее, $a^{*}$ есть произвольный вектор в $\Pi_{1}$ такой, что $\mathbf{P}\left\{\xi_{1}=a^{*}\right\}>$ 0 ; тогда искомая минимальная решетка $L^{\xi}$ имеет вид

$$
L^{\xi}=L_{m}(M, a)=a^{*}+\Gamma^{*},
$$


где матрица $M$ и вектор $a$ строятся по группе $\Gamma^{*}$ и вектору $a^{*}$ (см. (32)). Минимальная решетка $L_{m}(M, a)$ для вектора $\xi$ построена.

Завершим теперь доказательство леммы 1 . Умножая вектор $\xi$ справа на матрицу $M$, мы получаем $\xi M=a+\xi^{*}$, где координаты вектора $\xi^{*}$ образуют два класса: первый класс $\left(\xi_{1}^{*}, \ldots, \xi_{m}^{*}\right)$ составляет арифметический вектор с н.о.д. по каждой координате равным 1 , второй класс

$$
\left(\xi_{m+1}^{*}, \ldots, \xi_{d}^{*}\right)
$$

составляет нерешетчатый вектор. Этот второй класс и образует третыю группу объема $m_{3}=d-m$ в доказываемой лемме. Осталось разделить первый класс координат $\left(\xi_{1}^{*}, \ldots, \xi_{m}^{*}\right)$ на первую и вторую группы в доказываемой лемме: координата $\xi_{i}^{*}$ относится к первой группе, если . $\left\{a_{i}\right\}=0$, и относится ко второй группе, если $\left\{a_{i}\right\}>0, i=1,2, \ldots, m$ (напомним, что $\{t\}$ есть дробная часть числа $t$ ). Лемма 1 доказана.

2.3. Доказательство теоремы 1 . Воспользуемся следующим преобразованием меры $F$ (известное преобразование Крамера [3]):

$$
F^{(\alpha)}(A)=\frac{\mathbf{E}\left(e^{(\lambda(\alpha), \xi\rangle} ; \xi \in A\right)}{\varphi(\lambda(\alpha))}
$$

где $\lambda(\alpha)=\Lambda^{\prime}(\alpha), \alpha \in\left(A^{\prime}\right)$. Обозначим $\xi^{(\alpha)}(1), \xi^{(\alpha)}(2), \ldots$ последовательность независимых случайных векторов с общим распределением $F^{(\alpha)}$. Соответственно суммы таких векторов обозначим $S^{(\alpha)}(n)=$ $\xi^{(\alpha)}(1)+\cdots+\xi^{(\alpha)}(n)$. В этих обозначениях справедливо соотношение (см. [3])

$$
\begin{aligned}
& \mathbf{P}\left\{S(n) \in \Delta^{(m)}(n \alpha)\right\} \\
& \quad=e^{-n \Lambda(\alpha)} \mathbf{E}\left(e^{-\left\langle\lambda(\alpha), S^{(\alpha)}(n)-n \alpha\right\rangle} ; S^{(\alpha)}(n)-n \alpha \in \Delta^{(m)}(0)\right) .
\end{aligned}
$$

Поскольку для компакта $K \subseteq\left(A^{\prime}\right)$ верно $\lambda(K) \equiv \sup _{\alpha \in K}|\lambda(\alpha)|<\infty$, то справедливы следующие неравенства:

$$
\begin{aligned}
& \mathbf{P}\left\{S^{(\alpha)}(n)-n \alpha \in \Delta^{(m)}(0)\right\}\left(1-e^{-\lambda(K) \Delta d^{1 / 2}}\right) \\
& \quad \leqslant e^{n \Lambda(\alpha)} \mathbf{P}\left\{S(n) \in \Delta^{(m)}(n \alpha)\right\} \\
& \quad \leqslant \mathbf{P}\left\{S^{(\alpha)}(n)-n \alpha \in \Delta^{(m)}(0)\right\}\left(e^{\lambda(K) \Delta d^{1 / 2}}-1\right) .
\end{aligned}
$$

Утверждение теоремы 1 будет следовать из двух лемм.

Лемма 2. Для любого $\Delta>0$, любого компакта $K \subset\left(A^{\prime}\right)$ найдутся челое $n_{0}<\infty$ и вещественное $C<\infty$ такие, что для $n \geqslant n_{0}, \alpha \in K$ выполндется

$$
\mathbf{P}\left\{S^{(\alpha)}(n)-n \alpha \in \Delta^{(m)}(0)\right\} \leqslant \frac{C}{n^{d / 2}} .
$$


Лемма 3. Для любого $\Delta>0$, любого компакта $K \subset\left(A^{\prime}\right)$ найдутся челое $n_{0}<\infty$ и вещественное $c>0$ такие, что для $n \geqslant n_{0}, \alpha \in K$, $n \alpha \in \mathbf{R}^{d}(m)$, выполняется

$$
\mathbf{P}\left\{S^{(\alpha)}(n)-n \alpha \in \Delta^{(m)}(0)\right\} \geqslant \frac{c}{n^{d / 2}} .
$$

Доказательства лемм 2, 3 будут приведены в разделе 3.

Теорема 1 доказана.

2.4. Доказательство теоремы 2. I. Предположим сначала, что выполнено условие $\left(C_{1}\right)$, и докажем оценку сверху для верхнего предела в утверждении (9). В силу неравенства Чебышева справедливо (см., например, [3])

$$
\ln \mathbf{P}\{S(n) \in \Delta(n \alpha)\} \leqslant-\min _{\beta \in \Delta_{1}(\alpha)} \Lambda(\beta),
$$

где $\Delta_{1}=\Delta / n$. Поэтому оценка сверху в (9) будет следовать из соотношения

$$
\lim _{n \rightarrow \infty} \sup _{\alpha \in K}|\varepsilon(n, \Delta, \alpha)|=0,
$$

где $\varepsilon(n, \Delta, \alpha)=\Lambda(\alpha)-\min _{x \in \Delta_{1}(\alpha)} \Lambda(x)$. Для доказательства (36) выберем $\delta>0$ и компакт $K_{1} \subseteq(\Lambda)$ такие, что $U_{\delta}(K)=\{x:|x-y|<\delta, y \in K\} \subseteq$ $K_{1}$. Очевидно, далее, что если

$$
r(n) \equiv \frac{d^{1 / 2} \Delta}{n} \leqslant \delta
$$

то $\Delta_{1}(\alpha) \subseteq K_{1}$. Поэтому, если $r(n)$ удовлетворяет (37), то для $\alpha \in K$ выполняется неравенство

$$
|\varepsilon(n, \Delta, \alpha)| \leqslant \omega_{K_{1}}(r(n)),
$$

где $\omega_{K_{1}}(r)$ есть модуль непрерывности функции $\Lambda(x)$ на компакте $K_{1}$. Поскольку функция уклонений $\Lambda(x)$ на компакте $K_{1} \subseteq(\Lambda)$ равномерно непрерывна, то $\omega_{K_{1}}(r) \rightarrow 0$ при $r \rightarrow 0$; соотношение (36) доказано. Оценка сверху для верхнего предела в утверждении (9) установлена. Заметим попутно, что если условие $\left(C_{1}\right)$ не выполнено, то оценка сверху для верхнего предела в (10) тривиальна и не требует доказательства.

II. Пусть выполнено условие $\left(C_{1}^{(0)}\right)$; докажем утверждение $(11)$ теоремы 2. Заметим, что (см., например, [3])

$$
\mathbf{P}\{|S(n)| \geqslant n T\} \leqslant \mathbf{P}\left\{\sum_{k=1}^{n}|\xi(k)| \geqslant n T\right\} \leqslant \exp \left\{-n \inf _{t \geqslant T} \Lambda_{1}(t)\right\},
$$

где $\Lambda_{1}(t)=\sup _{u}\left\{t u-\ln \mathbf{E} e^{u|\xi|}\right\}$ есть функция уклонений случайной величины $|\xi|$. Поскольку при выполнении условия $\left(C_{1}^{(0)}\right)$ справедливо (см. [3]) $\lim _{T \rightarrow \infty} \inf _{t \geqslant T} \Lambda_{1}(t)=\infty$, то соотношение (11) доказано. 
III. Докажем оценку снизу в утверждениях (9), (10) теоремы 2. Пусть случайная величина $\gamma \geqslant 1$ не зависит от вектора $\boldsymbol{\xi}$ и $\mathbf{E} e^{u \gamma}<\infty$ для всех $u$. Для произвольного $1 \leqslant N \leqslant \infty$ введем новый случайный вектор $\xi_{(N)}$ с распределением

$$
F_{(N)}(V)=\mathbf{P}\left\{\xi_{(N)} \in V\right\} \equiv \mathbf{P}\{\xi \in V|| \xi \mid \leqslant N \gamma\}=\frac{\mathbf{P}\{\xi \in V ;|\xi| \leqslant N \gamma\}}{\mathbf{P}\{|\xi| \leqslant N \gamma\}} .
$$

Поскольку носители распределений $F$ и $F_{(N)}$ совпадают, то векторы $\xi$ и $\xi_{(N)}$ имеют одинаковые минимальные решетки. Иначе говоря, процедура построение вектора $\xi_{(N)}$ с помощью случайного уровня $N \gamma$ не меняет структуры исходного вектора $\xi$, в то время, как та же процедура с не случайным уровнем $N$ может привести к другой структуре вектора $\xi_{(N)}$ (скажем, превратить нерешетчатую часть исходного вектора в решетчатую). С другой стороны, вектор $\xi_{(N)}$ обладает следующим свойством, которого может не быть у исходного вектора $\xi$ : для любых $N \geqslant 1, \lambda \in \mathbf{R}^{d}$

$$
\varphi_{(N)}(\lambda) \equiv \mathbf{E}^{\left\langle\lambda, \xi_{(N)}\right\rangle}<\infty .
$$

Обозначим далее $\xi_{(N)}(1), \xi_{(N)}(2), \ldots$ независимые случайные векторы с общим распределением $F_{(N)}$; суммы таких векторов обозначим $S_{(N)}(n)=\xi_{(N)}(1)+\cdots+\xi_{(N)}(n)$. Все обозначения, отвечающие распределению $F_{(N)}$, снабдим нижним индексом $(N)$; например,

$$
\begin{aligned}
\varphi_{(N)}(\lambda) & =\mathbf{E} e^{\left(\lambda, \xi_{(N)}\right\rangle}, \quad \lambda \in \mathbf{R}^{d} ; \\
\Lambda_{(N)}(\alpha) & =\sup _{\lambda}\left\{\langle\lambda, \alpha\rangle-\ln \varphi_{(N)}(\lambda)\right\}, \quad \alpha \in \mathbf{R}^{d} .
\end{aligned}
$$

Для произвольного $N<\infty$ справедливы неравенства

$$
\begin{aligned}
\frac{1}{n} & \ln \mathbf{P}\{S(n) \in \Delta(n \alpha)\} \\
& \geqslant \frac{1}{n} \ln \mathbf{P}\{S(n) \in \Delta(n \alpha),|\xi(i)| \leqslant N \gamma, i=1, \ldots, n\} \\
& \geqslant \frac{1}{n} \ln \mathbf{P}\left\{S_{(N)}(n) \in \Delta(n \alpha)\right\}+\ln \mathbf{P}\{|\xi(1)| \leqslant N \gamma\} .
\end{aligned}
$$

В силу (38) область $\left(A_{(N)}^{\prime}\right)$ аналитичности функции $\Lambda_{(N)}(\alpha)$ совпадает с множеством $\left(\Lambda_{(N)}\right)=(\Lambda)$. Поэтому компакт $K$ лежит в области $\left(A_{(N)}^{\prime}\right)$, и мы можем воспользоваться теоремой 1 , в силу которой существуют константы $b_{1}=b_{1}(N, K)>0, n_{0}=n_{0}(N, K)<\infty$ такие, что для $\alpha \in K, n \alpha \in \mathbf{R}^{d}(m), n \geqslant n_{0}$ выполняется

$$
\frac{1}{n} \ln \mathbf{P}\left\{S_{(N)}(n) \in \Delta(n \alpha)\right\} \geqslant-\Lambda_{(N)}(\alpha)-\frac{1}{n} \ln \left(b_{1}(N) n^{d / 2}\right) .
$$

Поэтому, в силу (39), получаем для $\alpha \in K, n \alpha \in \mathbf{R}^{d}(m)$

$$
\frac{1}{n} \ln \mathbf{P}\{S(n) \in \Delta(n \alpha)\} \geqslant-\Lambda(\alpha)-\varepsilon_{1}-\varepsilon_{2}-\varepsilon_{3},
$$


где

$$
\begin{aligned}
& \varepsilon_{1}=\varepsilon_{1}(N) \equiv \ln \mathbf{P}\{|\xi| \leqslant N \gamma\}, \quad \varepsilon_{2}=\varepsilon_{2}(N) \equiv \sup _{\alpha \in K}\left|\Lambda_{(N)}(\alpha)-\Lambda(\alpha)\right|, \\
& \varepsilon_{3}=\varepsilon_{3}(N, n) \equiv \frac{1}{n} \ln \left(b_{1}(N) n^{d / 2}\right) .
\end{aligned}
$$

Очевидно, что $\lim _{N \rightarrow \infty} \varepsilon_{1}(N)=0$. Поскольку для любого $\lambda \in \mathbf{R}^{d}$ $\lim _{N \rightarrow \infty} \varphi_{(N)}(\lambda)=\varphi(\lambda)$, то в том случае, когда выполнено условие $\left(C_{1}\right)$, в силу теоремы 1 в [3] для любого $\alpha \in(\Lambda)$ имеет место сходимость функций уклонений

$$
\lim _{N \rightarrow \infty} \Lambda_{(N)}(\alpha)=\Lambda(\alpha) .
$$

Если же условие $\left(C_{1}\right)$ не выполнено, то мы не можем воспользоваться упомянутой теоремой 1 в [3], поскольку это условие присутствует в ней. В этом случае для любого $\lambda \in \mathbf{R}^{d}, \lambda \neq 0$, выполняется $\lim _{N \rightarrow \infty} \varphi_{(N)}(\lambda)=\infty$, поэтому сходимость (40) в форме $\lim _{N \rightarrow \infty} \Lambda_{(N)}(\alpha)=0$ тоже имеет место. Из $(40)$, в силу выпуклости функций $\Lambda_{(N)}(\alpha), \Lambda(\alpha)$, вытекает сходимость $\lim _{N \rightarrow \infty} \varepsilon_{2}(N)=0$. Поскольку $\lim _{n \rightarrow \infty} \varepsilon_{3}(N, n)=0$, для любого фиксированного $N$, то найдется функщия $N=N(n)$, стремящаяся к $\infty$ достаточно медленно, для которой

$$
\lim _{n \rightarrow \infty}\left(\varepsilon_{1}(N(n))+\varepsilon_{2}(N(n))+\varepsilon_{3}(N(n), n)\right)=0 .
$$

Оценки снизу для нижних пределов в утверждениях (9), (10) доказаны; теорема 2 доказана.

Поскольку доказательства теорем 3,4 (на базе уже доказанньх теорем 1,2 ) практически не отличаются от доказательств теорем 4,5 и 6 в [2], то мы их опускаем.

\section{3. Доказательство вспомогательных лемм}

\section{1. Доказательство леммы 2. Обозначим}

$$
\chi^{(\alpha)}(u)=\inf _{|\lambda|=1} \mathbf{E}\left(\left\langle\lambda, \xi^{(\alpha)}-\xi^{(\alpha)^{\prime}}\right\rangle^{2} ;\left|\left\langle\lambda, \xi^{(\alpha)}-\xi^{(\alpha)^{\prime}}\right\rangle\right|<u\right)
$$

где случайные векторы $\xi^{(\alpha)}, \xi^{(\alpha)^{\prime}}$ независимы и имеют общее распределение $F^{(\alpha)}$;

$$
N^{(\alpha)}(\Delta)=\sup _{u \geqslant \Delta} \frac{\chi^{(\alpha)}(u)}{u^{2}}
$$

Непосредственно из следствия из теоремы 6.2 в [13] вытекает утверждение: существует константа $C(d)$, зависяиая только от $d$, тахая, что для всех $n \geqslant 1, \alpha \in \mathbf{R}^{d}$ выполняется неравенство

$$
\mathbf{P}\left\{S^{(\alpha)}(n)-n \alpha \in \Delta^{(m)}(0)\right\} \leqslant \frac{C(d)}{n^{d / 2}} N^{(\alpha)}(\Delta)
$$


Поэтому лемма 2 будет слецовать из неравенства

$$
\sup _{\alpha \in K} N^{(\alpha)}(\Delta)<\infty
$$

Докажем (41). Легко видеть, что для $u>0 \chi^{(\alpha)}(u) \leqslant 2 \mathbf{E}\left|\xi^{(\alpha)}\right|^{2}$, поэтому

$$
N^{(\alpha)}(\Delta) \leqslant \frac{2 \mathbf{E}\left|\xi^{(\alpha)}\right|^{2}}{\Delta^{2}}
$$

Следуюшее утверждение будет доказано несколько позже, в п. 3.4.

Лемма 4. Для любого компакта $K \subset\left(A^{\prime}\right)$ найдется число $C<\infty$ такое, что для $\alpha \in K$ выполияется

$$
\mathbf{E}\left|\xi^{(\alpha)}\right|^{2} \leqslant C
$$

В силу (42) для любого $\alpha \in G_{1}$ выполняется $N^{(\alpha)}(\Delta) \leqslant 2 C / \Delta^{2}$. Соотношение (41) установлено; лемма 2 доказана.

3.2. Доказательство леммы 3. Введем, следуя [14], для любых векторов $b, r \in \mathbf{R}^{d}(m)$ числа

$$
\begin{aligned}
D_{n}(\alpha, b, r)= & \mathbf{P}\left\{S^{(\alpha)}(n)-n \alpha \in b+\Delta_{0}^{(m)}(0)\right\} \\
& -\mathbf{P}\left\{S^{(\alpha)}(n)-n \alpha \in b+r+\Delta_{0}^{(m)}(0)\right\} .
\end{aligned}
$$

Следующее утверждение, которое будет доказано в п. 3.3 , является аналогом леммы 3 [14].

Лемма 5. Для любого $\Delta>0$, любого компакта $K \subset\left(A^{\prime}\right)$ найдется число $C_{0}<\infty$ такое, что выполняется

$$
\sup _{\alpha \in K, n \alpha \in \mathbf{R}^{d}(m)} \sup _{b \in \mathbf{R}^{d}(m)}\left|D_{n}(\alpha, b, r)\right| \leqslant \frac{C_{0}|r| / \sqrt{n}+\varepsilon_{n}(r)}{n^{d / 2}},
$$

гдe $\lim _{n \rightarrow \infty} \sup _{r \in \mathbf{R}^{d}(m)}\left|\varepsilon_{n}(r)\right|=0$.

Покажем, как с помощью леммы 5 доказать лемму 3. Для этого нам потребуется следующее утверждение, которое будет доказано в п. 3.4.

Лемма 6. Для любого $\Delta>0$, любого компакта $K \subset\left(A^{\prime}\right)$ найдется число $c>0$ такое, что для $0<\tau \leqslant 1$ выполняется

$$
\liminf _{n \rightarrow \infty} \inf _{\alpha \in K} \mathbf{P}\left\{S^{(\alpha)}(n)-n \alpha \in \tau \sqrt{n} \Delta(0)\right\} \geqslant \tau^{d} c .
$$

В силу (44) для любого $0<\tau<1$ найдется последовательность векторов $x(n, \alpha) \in \mathbf{R}^{d},|x| \leqslant \tau \Delta \sqrt{d n}$, для которой выполняется

$$
\liminf _{n \rightarrow \infty} \inf _{\alpha \in K} \mathbf{P}\left\{S^{(\alpha)}(n)-n \alpha \in x(n, \alpha)+\Delta(0)\right\} \geqslant \frac{c}{n^{d / 2}} .
$$


Пусть теперь $\Delta<1$. Тогда очевидно, что для любого $0<\tau<1$ найдется последовательность векторов $x^{\prime}(n, \alpha) \in \mathbf{R}^{d}(m),\left|x^{\prime}\right| \leqslant\left((\tau \Delta \sqrt{d n})^{2}+d^{2}\right)^{1 / 2}$, для которой выполняется

$$
\liminf _{n \rightarrow \infty} \inf _{\alpha \in K} \mathbf{P}\left\{S^{(\alpha)}(n)-n \alpha \in x^{\prime}(n, \alpha)+\Delta_{0}^{(m)}(0)\right\} \geqslant \frac{c}{n^{d / 2}}
$$

Тогда для $\Delta<1$ и любого $0<\tau<1$ в силу (43), (45) найдется целое $n_{0}=n_{0}(\tau, \Delta)<\infty$ такое, что для всех $n \geqslant n_{0}$ справедливо

$$
\begin{aligned}
\mathbf{P} & \left\{S^{(\alpha)}(n)-n \alpha \in \Delta_{0}^{(m)}(0)\right\} \\
& \geqslant \mathbf{P}\left\{S^{(\alpha)}(n)-n \alpha \in x^{\prime}(n, \alpha)+\Delta_{0}^{(m)}(0)\right\}-D_{n}\left(\alpha, 0, x^{\prime}(n, \alpha)\right) \\
& \geqslant \frac{c-C_{0}\left((\tau \Delta \sqrt{d n})^{2}+d^{2}\right)^{1 / 2} / \sqrt{n}-\varepsilon_{n}}{n^{d / 2}} .
\end{aligned}
$$

Поэтому, выбирая $\tau>0$ достаточно малым, получаем утверждение леммы 3.

3.3. Доказательство леммы 5. Обозначим $k=d-m$ число нерешетчатых координат вектора $\xi$. Для $x \in \mathbf{R}^{d}$ через $x_{(1)} \in \mathbf{R}^{d}$ обозначим проекцию этого вектора на подпространство, порожденное векторами $e_{1}, \ldots, e_{m}$. Иначе говоря, для $x=\left(x_{1}, \ldots, x_{d}\right) \in \mathbf{R}^{d}$ выполняется $x_{(1)}=$ $\left(x_{(1) 1}, \ldots, x_{(1) d}\right), x_{(1) j}=x_{j}$ при $1 \leqslant j \leqslant m, x_{(1) j}=0$ при $m+1 \leqslant j \leqslant d$. Аналогично определим $x_{(2)}=x-x_{(1)}: x_{(2)}=\left(x_{(2) 1}, \ldots, x_{(2) d}\right), x_{(2) j}=0$ при $1 \leqslant j \leqslant m, x_{(2) j}=x_{j}$ при $m+1 \leqslant j \leqslant d$.

Соответственно, обозначим

$$
\mathbf{R}_{(s)}^{d}=\left\{x \in \mathbf{R}^{d}: x=x_{(s)}\right\}, \quad s=1,2 .
$$

Для произвольного $\varepsilon>0$ рассмотрим (см. доказательство леммы 3 в [14]) случайный вектор $\eta_{\varepsilon} \in \mathbf{R}_{(2)}^{d}$ такой, что $\mathbf{P}\left\{\left|\eta_{\varepsilon}\right| \leqslant \varepsilon\right\}=1$ и для соответствуюшей этому вектору характеристической функции $f_{\varepsilon}\left(t_{(2)}\right)=$ $\mathbf{E} e^{\left\langle t_{(2)}, \eta_{\varepsilon}\right\rangle}$ при $k=d-m \geqslant 1$ выполняется неравенство

$$
\left|f_{\varepsilon}\left(t_{(2)}\right)\right| \leqslant \frac{c}{\varepsilon^{k+1}\left|t_{(2)}\right|^{k+1}},
$$

где $c$ не зависит от $t_{(2)}$ и $\varepsilon$. Вектор $\eta_{\varepsilon}$ будет сглаживать «нерешетчатую» часть $S_{(2)}(n)-n \alpha_{(2)}$ вектора $S(n)-n \alpha$ при $n \alpha \in \mathbf{R}^{d}(m)$.

Отметим, что для $x \in \mathbf{R}^{d}(m)$ выполняется $x_{(1)} \in \mathbf{R}_{(1)}^{d}(m)$, где $\mathbf{R}_{(1)}^{d}(m)=\mathbf{Z}^{d} \cap \mathbf{R}_{(1)}^{d} ;$ соответственно, $x_{(2)} \in \mathbf{R}_{(2)}^{d}$. В пространстве $\mathbf{R}^{d}(m)$ через $\nu=\nu(A)$ обозначим меру, определяемую соотношением:

$$
\nu(d x)=\nu_{(1)}\left(d x_{(1)}\right) \nu_{(2)}\left(d x_{(2)}\right)
$$

где $\nu_{(1)}$ - считающая мера в $\mathbf{R}_{(1)}^{d}(m), \nu_{(2)}$ - мера Лебега в $\mathbf{R}_{(2)}^{d}$. Для $n \alpha \in \mathbf{R}^{d}(m)$ введем теперь плотность распределения случайного вектора 
$S^{(\alpha)}(n)-n \alpha+\eta_{\varepsilon}$ относительно меры $\nu$ :

$$
p\left(S^{(\alpha)}(n)-n \alpha+\eta_{\varepsilon} ; x\right)=\frac{\mathbf{P}\left\{S^{(\alpha)}(n)-n \alpha+\eta_{\varepsilon} \in d x\right\}}{\nu(d x)}
$$

в точке $x \in \mathbf{R}^{d}(m)$. Будем использовать следующее представление для этой плотности:

$$
\begin{aligned}
& p\left(S^{(\alpha)}(n)-n \alpha+\eta_{\varepsilon} ; x\right) \\
& \quad=\frac{1}{(2 i \pi)^{d}} \int_{[-\pi, \pi]^{m} \times \mathbf{R}^{k}} e^{-i\langle t, x\rangle} f^{n}(\alpha ; t) e^{-i\langle t, n \alpha\rangle} f_{\varepsilon}(t) d t
\end{aligned}
$$

где $f(\alpha ; t)=\mathbf{E} e^{i\left\langle t, \xi^{(\alpha)}(1)\right\rangle},[-\pi, \pi]^{m} \times \mathbf{R}^{k}=\left\{t=\left(t_{1}, \ldots, t_{d}\right) \in \mathbf{R}^{d}: t_{j} \in\right.$ $\left.[-\pi, \pi], 1 \leqslant j \leqslant m, t_{j} \in \mathbf{R}^{1}, m+1 \leqslant j \leqslant d\right\}$.

Формула (46) получается, если рассмотреть производяшую функцию по «решетчатым» координатам и характеристическую функцию по остальньм координатам и сначала написать формулу обрашения для сглаженной характеристической функции (это будет интеграл по $\mathbf{R}^{k}$ ), a затем обратить производящую функцию (для чего потребуется интеграл по компакту $\left.[-\pi, \pi]^{m}\right)$.

Следующее утверждение будет доказано в п. 3.4.

Лемма 7. Для любого компакта $K \subset\left(A^{\prime}\right)$, пюбого $t \in[-\pi, \pi]^{m} \times$ $\mathbf{R}^{k}, t \neq 0$, выполняется $\sup _{\alpha \in K}|f(\alpha ; t)|<1$.

Далее доказательство леммы 5 (с использованием представления (46) и леммы 7) является практически дословным повторением доказательства леммы 3 в [14], поэтому мы его опускаем. Лемма 5 доказана.

3.4. Доказательство лемм 4, 6, 7. Д ок а з а т ль с т в о л е м м ы 4 . Очевидно, что для любого компакта $K \subset\left(A^{\prime}\right)$ найдется $\delta>0$ такое, что

$$
\sup _{\alpha \in K} \mathbf{E} e^{|\delta|\left|\xi^{(\alpha)}(1)\right|}<\infty
$$

Из (47) следует утверждение леммы 4.

Доказательст во лем мы 6. Из (47) следует

$$
\sup _{\alpha \in K} \mathbf{E}\left|\xi^{(\alpha)}(1)-\alpha\right|^{3}<\infty
$$

Кроме того, в силу известных свойств функции уклонений (см., например, [3]), для $\alpha \in\left(A^{\prime}\right)$ выполняется

$$
\mathbf{E} \xi^{(\alpha)}=\alpha, \quad B(\alpha) \equiv \mathbf{E}\left(\xi^{(\alpha)}-\alpha\right)^{T}\left(\xi^{(\alpha)}-\alpha\right)=\left(\Lambda^{\prime \prime}(\alpha)\right)^{-1}
$$

поэтому для $\sigma^{2}(\alpha)=\operatorname{det}(B(\alpha))$ справедливо

$$
\inf _{\alpha \in K} \sigma(\alpha)>0
$$


Из соотношений (48), (49), в силу известньх оценок скорости сходимости в центральной предельной теореме, получаем утверждение леммы 6.

Д ок аз ательст во лем мы 7. Очевидно, что для любого $t \in[-\pi, \pi]^{m} \times \mathbf{R}^{k}, t \neq 0$, выполняется $|f(\alpha ; t)|<1$. Поэтому утверждение леммы 7 является непосредственным следствием леммы 8 в [3] (с. 56).

В заключение авторы выражают благодарность А. Ю. Зайцеву, который обратил наше внимание на работу [14], а также рецензенту, который указал нам на работы [4]-[8].

\section{СПИСОК ЛИТЕРАТУРЫ}

1. Боровков A.A., Могульский $A . A$. Интегро-локальные предельные теоремы для сумм случайных векторов, включающие большие уклонения. I. - Теория вероятн. и ее примен., 1998, т. 43, № 1, с. 3-17.

2. Боровков A. A., Могульский $A$. A. Вторая функшия уклонений и асимптоттгеские задачи восстановления и достижения границы для многомерных блужданий. - Сиб. матем. журн., 1996 , т. 37, Nㅡㄴ, 4, 745-782.

3. Боровков A. A., Могульский $A$. A. Большие уклонения и проверка статистических гипотез. Новосибирск: Наука, 1992, 222 с.

4. von Barh $B$. Multi-dimensional integral limit theorem for large deviations. - Ark. Mat., 1967, v. 7, p. 89-99.

5. Осипов Л.В. Вероятности больших уклонений сумм независимых случайных вехторов для некоторых классов множеств. - Матем. заметки, 1982, т. 31, № 1 , c. $147-155$.

6. Osipov $L$. V. On large deviations for sums of random vectors in $R^{k}$. - J. Multivariate Anal., 1981, v. 11, p. 115-126.

7. Алешхявичене $A . K$. Многомерные интегральные предельные теоремы для вероятностей больших уклонений. - Теория вероятн. и ее примен., 1983, т. 28, в. 3 , c. $62-82$.

8. Алешкявичене $A$., Светуляничене $B$. Вероятности умеренно больших уклонений в многомерном случае. - Литов. матем. журн., 1984, т. 24, с. 201-209.

9. Бхаттачария $P$. H., Ранга $P$ aо $P$. Аппроксимация нормальным распределением и асимптотические разложения. М.: Наука, 1982, 286 с.

10. Bahadur R. R., Ranga Rao R. On deviations of the sample mean. - Ann. Math. Statist., 1960, v. 31, № 4, p. 1015-1027.

11. Петров В.В. О вероятностях больших уклонений сумм независимых случайных величин. - Теория вероятн., и ее примен., 1965, т. 10, в. 2, с. 310-322.

12. Dinwood $I$. $H$. A note on the upper bound for i.i.d. large deviations. - Ann. Probab., 1991 , v. 19, № 2, p.1732-1736.

13. Esseen $C$. G. On the concentration function of a sum of independent random variables. - Z. Wahrscheinlichkeitstheor. verw. Geb., 1968, B. 9, S. 290-308.

14. Мухия $A . B$. О лохальных вероятностях сумм независимых случайных величия. - Теория вероятн. и ее примен., 1989 , т. 34, в. 4, с. 677-685.

Поступила в редакцию 12.II.1999 